Doi: $10.32481 /$ djph.2021.09.023

\title{
Now is the Time for Delaware Families to Catch Up on Missed Immunizations
}

\author{
Kate Smith, MD, MPH \\ Program Manager, Immunization Coalition of Delaware
}

On August 23, 2021, the United States Food and Drug Administration (FDA) granted full approval to the first COVID-19 vaccine, Comirnaty (the vaccine developed by PfizerBioNTech), and President Biden urged those who were waiting for full approval of a COVID-19 vaccine to get one as soon as possible.

This national focus on increasing COVID-19 vaccination rates provides an important reminder of the role of immunizations in protecting Delawareans and their families from a range of other preventable illnesses. Unfortunately, the COVID-19 pandemic-related stay-at-home orders and safety precautions led many Delaware families to delay routine health care services, including well-child visits and vaccinations. This pause has left children, adolescents, and adults (including pregnant women) vulnerable and at risk of contracting a broad range of other dangerous illnesses.

Recent data from the U.S. Centers for Disease Control and Prevention (CDC) highlight that across the country, rates of administered doses of routine childhood and adolescent vaccines were substantially lower from March to May 2020 in comparison to the years prior, and the Delaware Division of Public Health recognizes that many children are behind on their vaccinations. Under-vaccination reopens the door to many diseases that were thought to be under control or eradicated, including measles, mumps, meningitis, or whooping cough.

Back-to-school season is an important time for families to get caught up on all appropriate vaccinations, especially since Delaware public schools require all children to receive vaccines for certain preventable diseases as a condition of enrollment. This is a seemingly small step that will have an enormous impact. Before the measles vaccine was available in 1963, almost every child became infected before age 15 and over 500 people died each year. Now, most physicians go their entire career without treating a single case. As a result of broad and effective childhood immunizations, children rarely die from what were once common illnesses.

Preventing disease outbreaks, especially as our health care resources are being stretched thin by COVID-19 and the Delta variant, is paramount. Hundreds of thousands of people are hospitalized with the flu each year, and tens of thousands die. The flu vaccine is approved for everyone over the age of six months, and is the best way to help protect against the flu, keep people out of hospitals, and prevent outbreaks.

On August 20, Governor Carney signed a proclamation stating that August was Immunization Awareness Month in Delaware. Parents and guardians take steps every day to keep their children healthy and safe: using car seats, holding hands when crossing the street, making sure everyone eats a healthy diet. Along with the National Immunization Awareness Campaign, the Immunization Coalition of Delaware urges you to get your flu shot, get your COVID-19 vaccine and booster, and have a conversation with your doctor today about catching up on appropriate vaccines for every member of your family. 
Doi: 10.32481/djph.2021.09.023

Copyright (c) 2021 Delaware Academy of Medicine / Delaware Public Health Association.

This is an Open Access article distributed under the terms of the Creative Commons Attribution Non-Commercial License (https://creativecommons.org/licenses/by-nc-nd/4.0/) which permits unrestricted non-commercial use, distribution, and reproduction in any medium, provided the original work is properly cited. 